\title{
Statin use is associated with a reduced risk of hepatocellular carcinoma recurrence after initial liver resection
}

\author{
Yoshikuni Kawaguchi $^{1}$, Yoshihiro Sakamoto ${ }^{1}$, Daisuke Ito ${ }^{1}$, Kyoji Ito ${ }^{1}$, Junichi Arita ${ }^{1}$, \\ Nobuhisa Akamatsu', Junichi Kaneko ${ }^{1}$, Kiyoshi Hasegawa ${ }^{1}$, Kyoji Moriya ${ }^{2}$, \\ Norihiro Kokudo ${ }^{1,3, *}$ \\ ${ }^{1}$ Hepato-Biliary-Pancreatic Surgery Division, Department of Surgery, Graduate School of Medicine, The University of Tokyo, \\ Tokyo, Japan; \\ ${ }^{2}$ Department of Infection Control and Prevention, Graduate School of Medicine, The University of Tokyo, Tokyo, Japan; \\ ${ }^{3}$ National Center for Global Health and Medicine, Tokyo, Japan.
}

\begin{abstract}
Summary $\quad$ Effective adjuvant therapies have not been established for hepatocellular carcinoma (HCC). The study aimed to determine prognostic influence of statin against $\mathrm{HCC}$ recurrence after initial resection. From 2003 to 2013, 734 patients underwent initial HCC resection. Exposure to statins was defined as the use at the recommended daily dosage for $>90$ days after surgery. Outcomes were compared between patients who did and did not receive statins. Of 734 patients, $31(4.2 \%)$ received statins for dyslipidemia (statin group) and $703(95.8 \%)$ did not (non-statin group). The proportions of hepatitis B $(6.5 \%$ vs. $22.8 \%, P=0.032), C(19.4 \%$ vs. $45.0 \%, P=0.005)$, and a fibrosis score of F3-4 $(16.1 \% v s .39 .8 \%, P=0.008)$ were significantly lower in the statin than non-statin group. The recurrence-free survival rate was significantly higher in the statin than non-statin group $(P<0.001)$, without significant difference of the overall survival rate $(P=\mathbf{0 . 1 4 2})$. A multivariable Cox proportional hazards model revealed that the use of statins (hazard ratio, $0.34 ; P=0.005$ ) was associated with a significantly lower risk of HCC recurrence. After one-to-two propensity score matching, the RFS rate was also significantly higher in the statin group $(n=31)$ than in the non-statin group $(n=62)(P=0.008)$. In conclusion: The statins use reduced the risk of $\mathrm{HCC}$ recurrence after initial resection. Statins may have protective influences on $\mathrm{HCC}$ recurrence in patients who undergo initial liver resection.
\end{abstract}

Keywords: Statin, liver resection, hepatocellular carcinoma

\section{Introduction}

Hepatocellular carcinoma (HCC) is the fifth most common cancer worldwide and the second among causes of cancer-related deaths among men, whereas among women, it is the seventh most common cancer and the sixth leading cause of cancer death (1). Liver resection remains the optimal treatment for $\mathrm{HCC}$. Previously reported overall survival rates are $40 \%$ to

Released online in J-STAGE as advance publication October 29, 2017.

*Address correspondence to:

Dr. Norihiro Kokudo, National Center for Global Health and Medicine, 1-21-1 Toyama, Shinjuku-ku, Tokyo 162-8655, Japan. E-mail: nkokudo@hosp.ncgm.go.jp
$80 \%$ at 3 years and $20 \%$ to $70 \%$ at 5 years after $\mathrm{HCC}$ resection (2-4). Cumulative recurrence rates remain high $(50-60 \%$ at 3 years and $70-100 \%$ at 5 years) (2$6)$. Adjuvant therapy has been expected to reduce HCC recurrence and prolong postsurgical survival. Indeed, several drugs including interferon, sorafenib, and acyclic retinoid have been examined to determine their protective effect against HCC recurrence (7-10). However, effective adjuvant therapies for use after HCC resection have yet to be established.

The protective effects of statins against the development of $\mathrm{HCC}$ were recently indicated in patients with hepatitis $\mathrm{B}(11-13)$ and hepatitis $\mathrm{C}$ $(14,15)$, although conflicting results have also been reported (16). Use of nucleoside analogues, statins, and nonsteroidal anti-inflammatory drugs (NSAIDs)/ 
aspirin were reportedly associated with a reduced risk of HCC recurrence in patients with hepatitis $\mathrm{B}$, who underwent liver resection (17). However, few studies have focused on the effectiveness of statin use against HCC recurrence after liver resection. We hypothesized that statin use can influence HCC recurrence after liver resection even in patients who receive statins on a daily basis for the treatment of dyslipidemia.

The aim of the present study was to determine the prognostic influence of statin use on initial liver resection of HCC by comparing long-term outcomes between patients who did and did not receive statins.

\section{Methods}

\subsection{Study population}

From January 2003 to December 2013, a total of 1,337 consecutive patients with HCC underwent liver resection at the University of Tokyo Hospital. The collected data were retrieved from prospectively maintained databases and included baseline patient characteristics such as specific drug use (statins), operative characteristics, histopathological data, and postoperative outcomes. Exposure to statins was defined as the use of statins at the recommended daily dosages for $>90$ days after surgery. These recommended daily dosages were as follows: pravastatin, $10 \mathrm{mg} /$ day; simvastatin, $5 \mathrm{mg}$ /day; fluvastatin, $20 \mathrm{mg} /$ day; pitavastatin, $1 \mathrm{mg}$ /day; atorvastatin, $5 \mathrm{mg} /$ day; and rosuvastain, $2.5 \mathrm{mg}$ /day. Patients who underwent repeated hepatectomy $(n=603)$ were excluded from the present study. The remaining 734 patients were included in the analysis. This study was conducted with the approval of the Institutional Ethics Review Board of The University of Tokyo (ID: 2158-5). Written informed consent was obtained from all patients.

\subsection{Surgical procedures and histopathological assessments}

Chest and abdominal contrast-enhanced computed tomography, and ultrasonography were routinely performed before surgical resection. Additionally, magnetic resonance imaging with Gd-EOB-DTPA (Bayer Schering Pharma, Berlin, Germany) had been performed since 2007. Liver resection was indicated according to specific criteria based on preoperative liver function parameters, such as the presence/absence of uncontrolled ascites, serum bilirubin concentration, and indocyanine green retention rate at $15 \mathrm{~min}$ (ICG-R15). $(18,19)$ Briefly, if the serum bilirubin ceoncentration was normal, our criteria permitted right hepatectomy or trisectoriectomy when the ICG-R15 was $<10 \%$, left hepatectomy or sectoriectomy when the ICG-R15 was $<20 \%$, subsegmentectomy or monosegmentectomy when the ICG-R15 was $<30 \%$, limited resection when the ICG-R15 was $<40 \%$, and enucleation when the ICG-R 15 was $>40 \%$.

The histologic classification of tumors and the background liver was based on the system established by the Liver Cancer Study Group of Japan (20). The histologic differentiation of HCC (well, moderate, or poor) was determined according to the Edmondson grade $(20,21)$. Both the fibrotic stage and the activity of hepatitis in the background liver were also recorded according to the classification proposed by Desmet et al. (22).

\subsection{Postoperative management}

Morbidity and mortality were defined as postoperative complications and death within 90 days after surgery, respectively. Postoperative morbidity was graded according to the Clavien-Dindo classification (23).

\subsection{Patient follow-up}

Measurement of blood tumor marker [alpha-fetoprotein (AFP) and des- $\gamma$-carboxyprothrombin (DCP)] were performed every month for six months after hospital discharge. Contrast-enhanced computed tomography or magnetic resonance imaging was performed every 3 to 4 months.

Recurrence was diagnosed as the appearance of a new lesion with radiographic features compatible with HCC. Recurrence-free survival (RFS) was defined as the interval between the operation and the date of diagnosis of the first recurrence, and overall survival (OS) was calculated based on the time from surgery to death or last follow-up.

\subsection{Statistical analysis}

Categorical variables are expressed as numerical figures (\%), and were compared between groups using Fisher's exact test or the chi-square test as appropriate. Continuous variables are expressed as median (interquartile range, IQR) and were compared using the Wilcoxon's rank-sum test. OS and RFS curves were constructed using the Kaplan-Meier method and compared using the log-rank test. Factors with a $\mathrm{P}$ value of $<0.10$ in a Cox proportional hazard model under univariable analysis were considered potential risk factors and were further analyzed in a multivariable Cox model. Hazard ratios (HRs) and 95\% confidence intervals (CIs) were calculated for each factor. Based on previously reported evidence, we chose potential confounders and dichotomized their cutoff levels of continuous variables $(5,24-30)$. A propensity scorematching analysis $(31,32)$ was used to build a matched group of patients. The propensity score model was estimated using a logistic regression model. A 1:2 match without replacement was performed using 
logit (propensity score) through the nearest available matching, setting the caliper at 0.20 . A $P$ value of $<$ 0.05 was considered to indicate statistical significance. Statistical analysis was conducted using JMP software (version 11.0; SAS Institute Inc., Cary, NC).

\section{Results}

\subsection{Patient characteristics}

Of the 734 patients, $31(4.2 \%)$ received statins for the treatment of dyslipidemia before surgery. There were no patients that received statin at the recommended daily dosages for $\leq 90$ days after surgery. The median statin administration period was $37.2(32.4-51.6)$ months postoperatively. The background characteristics were compared between patients who received statins (statin group) and patients who did not (non-statin group) (Table 1). The proportions of patients with hepatitis B surface antigen (HBsAg) positivity and hepatitis $\mathrm{C}$ virus antibody (HCVAb) positivity were significantly lower in the statin than non-statin group (HBsAg, 6.5\% vs. $22.8 \%, P=0.032$; HCVAb, $19.4 \%$ vs. $45.0 \%, P=0.005)$. The level of triglyceride was significantly higher in the statin groups than the non-statin group: [104 (81-150) vs. 89 (68-122) $\mathrm{mg} / \mathrm{dL}, P=0.030]$. The preoperative AFP concentration was significantly lower in the statin than non-statin group [3.5 (2.3-8.0) vs. 15.0 (5.0-65.3) ng/mL, $P<0.001]$. There were no significant differences in age, male/female ratio, proportion of patients with hepatitis B core antibody ( $\mathrm{HBcAb})$ positivity, Child-Pugh class/ score, number of tumors, maximum tumor diameter, liver functional parameters, or ICG-R15 between the two groups.

\subsection{Surgical, histopathological, and postoperative factors}

Surgical, histopathological, and postoperative factors are summarized in Table 2. Red blood cell transfusion were performed more frequently in the statin than nonstatin group $(16.1 \%$ vs. $3.1 \%, P=0.004)$. There were no significant differences in the operative time, estimated blood loss, or positive surgical margins between the two groups. With respect to histopathological factors, the proportion of patients with a fibrosis score of F3-4 was significantly lower in the statin than non-statin group (16.1\% vs. $39.8 \%, P=0.008)$. The proportions of tumor differentiation (poor) and major/minor vascular invasion were similar between the groups. The operative mortality rate was $0.0 \%$ in the statin group and $0.3 \%$ in the non-statin group $(P>0.999)$. The morbidity and major complication rates were similar between the groups. The postoperative hospital stay was significantly shorter in the statin than non-statin group [13 (10-16) days vs. 15 (12-19) days, $P=0.022]$.

\subsection{Patient survival}

The median follow-up time was 37.2 (32.4-51.6) months in the statin group and 31.2 (16.8-67.2) months in the non-statin group, demonstrating no significant difference $(P=0.598)$. The RFS rate was significantly higher in the statin than non-statin group $(P<0.001)$ : the 1-, 3-, and 5 - year RFS rates were $87.1 \%, 76.7 \%$, and $76.7 \%$, respectively, in the statin group, and $65.3 \%, 40.6 \%$, and $32.9 \%$, respectively, in the non-statin group (Figure 1). The OS rate was not significantly different between the groups: the 1-, 3-, and 5- year OS rates were 96.7\%,

Table 1. Patient demographic and clinical characteristics

\begin{tabular}{|c|c|c|c|}
\hline Variables & Statin group, $n=31$ & Non-statin group, $n=703$ & $P$ value \\
\hline Age, years & $68(64-75)$ & $67(59-73)$ & 0.233 \\
\hline Sex, male : female & $25: 6$ & $542: 161$ & 0.827 \\
\hline HBsAg, positive & $2(6.5)$ & $160(22.8)$ & 0.032 \\
\hline $\mathrm{HBcAb}$, positive & $8(25.8)$ & $239(34.0)$ & 0.439 \\
\hline HCVAb, positive & $6(19.4)$ & $316(45.0)$ & 0.005 \\
\hline Child-Pugh class ${ }^{*}, \mathrm{~A} / \mathrm{B} / \mathrm{C}$ & $28(90.3) / 3(9.7) / 0$ & $650(92.5) / 52(7.4) / 1(0.1)$ & 0.876 \\
\hline \multicolumn{4}{|l|}{ Medications } \\
\hline Statins ${ }^{\dagger}$ & $31(100)$ & - & - \\
\hline No. of tumors, multiple & $7(22.6)$ & $236(33.6)$ & 0.245 \\
\hline Maximum diameter of the tumors, $\mathrm{mm}$ & $32(18-106)$ & $37(23-60)$ & 0.461 \\
\hline AST, IU/L & $31(28-39)$ & $38(26-53)$ & 0.191 \\
\hline ALT, IU/L & $29(16-42)$ & $35(22-54)$ & 0.152 \\
\hline Total bilirubin, mg/dL & $0.7(0.5-0.8)$ & $0.7(0.5-0.9)$ & 0.494 \\
\hline Total cholesterol, mg/dL & $163(146-195)$ & $166(144-191)$ & 0.822 \\
\hline Triglyceride, mg/dL & $104(81-150)$ & $89(68-122)$ & 0.030 \\
\hline PT, $\%$ & $100.0(77.6-100.0)$ & $89.0(76.3-100)$ & 0.085 \\
\hline ICG-R15, \% & $10.5(6.4-16.7)$ & $12.1(8.0-18.9)$ & 0.207 \\
\hline AFP, ng/mL & $3.5(2.3-8.0)$ & $15.0(5.0-65.3)$ & $<0.001$ \\
\hline $\mathrm{DCP}, \mathrm{mAU} / \mathrm{mL}$ & $635.0(20.0-5076.0)$ & $102.0(22.0-1057.5)$ & 0.103 \\
\hline
\end{tabular}

Abbreviations: HBsAg, hepatitis B surface antigen; HBcAb, hepatitis B core antibody; HVCAb, hepatitis C virus antibody; AST, aspartate aminotransferase; ALT; alanine aminotransferase; PT, prothrombin time; ICG-R15, indocyanine green retention rate at 15 minutes; AFP, $\alpha$-fetoprotein; DCP, des- $\gamma$-carboxyprothrombin. ${ }^{*}$ Child-Pugh score, $5(5-6) v s .5(5-6), P=0.570 .{ }^{\dagger}$ Recommended dosages for statins: pravastatin, 10 mg/day $(n=5)$; simvastatin, $5 \mathrm{mg} /$ day $(n=1)$; pitavastatin, $1 \mathrm{mg} /$ day $(n=1)$; atorvastatin, $5 \mathrm{mg}$ /day $(n=19)$; and rosuvastain, $2.5 \mathrm{mg} / \mathrm{day}(n=5)$. 
Table 2. Surgical, histopathological, and postoperative factors

\begin{tabular}{lccc}
\hline Variables & Statin group, $n=31$ & Non-statin group, $n=703$ & $P$ value \\
\hline Surgical factors & & & 0.160 \\
$\quad$ Operative time, minutes & $317(228-457)$ & $356(275-460)$ & 0.059 \\
$\quad$ Estimated blood loss, $\mathrm{mL}$ & $430(210-1001)$ & $650(370-1144)$ & 0.004 \\
$\quad$ Red blood cell transfusion & $5(16.1)$ & $22(3.1)$ & 0.564 \\
Surgical margin, positive & $1(3.2)$ & $18(2.6)$ & 0.008 \\
Histopathological factors & $5(16.1)$ & $280(39.8)$ & 0.719 \\
$\quad$ Fibrosis score, F3-4* & $1(3.2)$ & $52(7.4)$ & 0.851 \\
Tumor differentiation, poor $\dagger$ & $11(35.5)$ & $271(38.6)$ & 0.271 \\
Vascular invasion & & & $>0.999$ \\
Postoperative factors & $4(12.9)$ & $162(23.0)$ & $>0.999$ \\
Morbidity & $1(3.2)$ & $2(0.3)$ & 0.022 \\
$\quad$ Major complication & 0 & $15(12-19)$ & \\
Mortality & $13(10-16)$ & & \\
Postoperative hospital stay, days & & & \\
\hline
\end{tabular}

*Based on the classification by Desmet et al. (22). †vs. well/moderate, based on modification of the Edmondson grade (21).

a

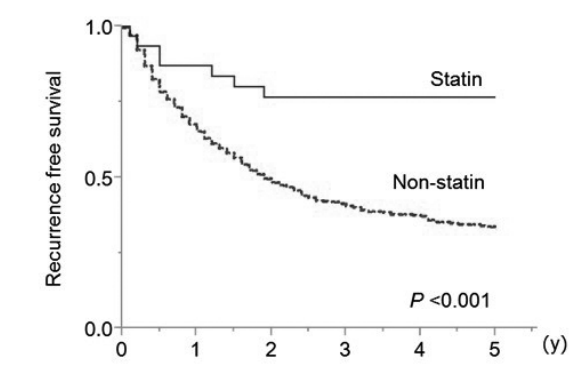

Patients at risk

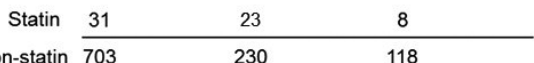

b

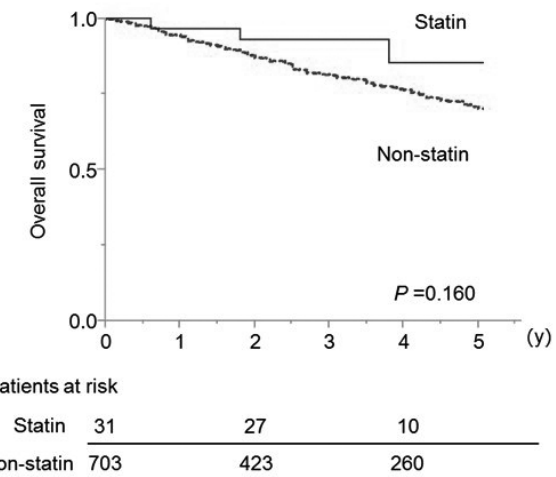

Figure 1. Long-term outcomes between the groups. (a) Recurrence-free survival rate was significantly higher in the statin than non-statin group $(P<0.001)$; (b) Overall survival rate was similar between the groups $(P=0.160)$.

$93.1 \%$, and $85.3 \%$, respectively, in the statin group, and $94.2 \%, 87.1 \%$, and $70.2 \%$, respectively, in the non-statin group $(P=0.142)$.

\subsection{Factors associated with recurrence and overall survival}

HCVAb positivity, use of statins, an ICG-R15 of $>15 \%$, a concentration of $>40 \mathrm{IU} / \mathrm{L}$, an AFP concentration of $>20$ $\mathrm{ng} / \mathrm{mL}$, a DCP concentration of $>40 \mathrm{mAU} / \mathrm{mL}$, multiple tumors, tumor size of $>2 \mathrm{~cm}$, and vascular invasion were found to be factors that potentially influenced RFS (Table 3). The multivariable Cox proportional hazards model revealed that use of statins (HR, 0.34; $95 \% \mathrm{CI}, 0.12-0.75$; $P=0.005$ ) was associated with a significantly lower risk of HCC recurrence. Additionally, an ICG-R15 of $>15 \%$ (HR, 1.27), ALT concentration of $>40 \mathrm{IU} / \mathrm{L}$ (HR, 1.46), AFP concentration of $>20 \mathrm{ng} / \mathrm{mL}$ (HR, 1.39), DCP concentration of $>40 \mathrm{mAU} / \mathrm{mL}$ (HR, 1.34), multiple tumors (HR, 1.94), tumor size of $>2 \mathrm{~cm}$ (HR, 1.38), and vascular invasion (HR, 1.22) were independent risk factors for RFS. Use of statins was not a significant risk factor for OS (HR, 0.44; 95\% CI, 0.11-1.15; $P=0.102$ ) (Table 4). HCVAb positivity (HR, 1.55), an ICG-R15 of $>15 \%$ (HR, 1.39), AFP concentration of $>20 \mathrm{ng} / \mathrm{mL}$ (HR, 1.37), multiple tumors (HR, 2.22), tumor size of $>$ $2 \mathrm{~cm}$ (HR, 1.72), poor tumor differentiation (HR, 1.79), and vascular invasion (HR, 1.47) were independent risk factors for OS.

\subsection{Patient survival evaluation using propensity score- matching analysis}

After 1:2 case propensity score matching, 31 patients in the statin group and 62 patients in the non-statin group were analyzed. Patient demographics, and surgical, histopathological, and postoperative outcomes were comparable after the matching (Supplementary Tables $\mathrm{S} 1$ and S2, http://www.biosciencetrends.com/action/ getSupplementalData.php? ID=15). The RFS rate was significantly higher in the statin than non-statin group ( $P$ $=0.008)$ (Figure 2$)$. The OS rate was not significantly different between the groups $(P=0.581)$.

\section{Discussion}

In the present study, the use of statins was significantly associated with a lower HCC recurrence rate. This finding is consistent with the RFS rates in the cohort after excluding patients with hepatitis B and C. The 
Table 3. Cox proportional hazards model analysis for recurrence free survival

\begin{tabular}{|c|c|c|c|c|c|c|}
\hline \multirow{2}{*}{ Variables } & \multicolumn{3}{|c|}{ Univariable Analysis } & \multicolumn{3}{|c|}{ Multivariable Analysis } \\
\hline & HR & $95 \% \mathrm{CI}$ & $P$ value & HR & $95 \% \mathrm{CI}$ & $P$ value \\
\hline Age $>65$ years & 0.92 & $0.76-1.12$ & 0.414 & & & \\
\hline Male & 0.86 & $0.69-1.08$ & 0.186 & & & \\
\hline HBsAg, positive & 1.06 & $0.84-1.34$ & 0.603 & & & \\
\hline $\mathrm{HBcAb}$, positive & 0.98 & $0.80-1.20$ & 0.866 & & & \\
\hline HCVAb, positive & 1.20 & $0.99-1.46$ & 0.062 & 1.05 & $0.85-1.30$ & 0.662 \\
\hline Fibrosis (F3-4) vs. (F0-2) & 1.11 & $0.91-1.35$ & 0.292 & & & \\
\hline Use of statin & 0.30 & $0.13-0.58$ & $<0.001$ & 0.32 & $0.11-0.70$ & 0.002 \\
\hline ICG-R $15>15 \%$ & 1.34 & $1.10-1.64$ & 0.004 & 1.27 & $1.02-1.58$ & 0.031 \\
\hline $\mathrm{ALT}>40 \mathrm{IU} / \mathrm{L}$ & 1.57 & $1.28-1.91$ & $<0.001$ & 1.46 & $1.18-1.80$ & $<0.001$ \\
\hline $\mathrm{AFP}>20 \mathrm{ng} / \mathrm{mL}$ & 1.66 & $1.37-2.02$ & $<0.001$ & 1.39 & $1.14-1.70$ & 0.001 \\
\hline $\mathrm{DCP}>40 \mathrm{mAU} / \mathrm{mL}$ & 1.39 & $1.14-1.71$ & 0.001 & 1.34 & $1.07-1.68$ & 0.009 \\
\hline Red blood cell transfusion & 0.84 & $0.40-1.53$ & 0.592 & & & \\
\hline Surgical margin, positive & 1.62 & $0.80-2.87$ & 0.165 & & & \\
\hline Multiple tumor vs. solitary tumor & 1.97 & $1.61-2.40$ & $<0.001$ & 1.94 & $1.58-2.38$ & $<0.001$ \\
\hline Tumor size $>2 \mathrm{~cm}$ & 1.49 & $1.15-1.97$ & 0.002 & 1.38 & $1.05-1.84$ & 0.022 \\
\hline Tumor differentiation, poor & 1.18 & $0.81-1.66$ & 0.384 & & & \\
\hline Vascular invasion, positive & 1.31 & $1.07-1.60$ & 0.008 & 1.22 & $0.99-1.49$ & 0.059 \\
\hline
\end{tabular}

Abbreviations: HBcAb, hepatitis B core antibody; HVCAb, hepatitis C virus antibody; ICG-R15, Indocyanine green retention rate at 15 minutes; ALT, alanine aminotransferase; AFP, $\alpha$-fetoprotein; DCP, des- $\gamma$-carboxyprothrombin.

Table 4. Cox proportional hazards model analysis for overall survival

\begin{tabular}{|c|c|c|c|c|c|c|}
\hline \multirow{2}{*}{ Variables } & \multicolumn{3}{|c|}{ Univariable Analysis } & \multicolumn{3}{|c|}{ Multivariable Analysis } \\
\hline & HR & $95 \% \mathrm{CI}$ & $P$ value & HR & $95 \% \mathrm{CI}$ & $P$ value \\
\hline Age $>65$ years & 1.21 & $0.90-1.64$ & 0.213 & & & \\
\hline Male & 0.80 & $0.58-1.09$ & 0.152 & & & \\
\hline HBsAg, postive & 0.69 & $0.46-1.00$ & 0.049 & 0.80 & $0.49-1.25$ & 0.329 \\
\hline $\mathrm{HBcAb}$, positive & 0.98 & $0.80-1.20$ & 0.866 & & & \\
\hline HCVAb, positive & 1.82 & $1.35-2.47$ & $<0.001$ & 1.55 & $1.08-2.24$ & 0.017 \\
\hline Fibrosis $(\mathrm{F} 3-4)$ vs. $(\mathrm{F} 0-2)^{*}$ & 1.13 & $0.84-1.52$ & 0.417 & & & \\
\hline Use of statin & 0.44 & $0.11-1.15$ & 0.102 & & & \\
\hline ICG-R15 > 15\% & 1.65 & $1.21-2.18$ & 0.001 & 1.39 & $1.01-1.92$ & 0.045 \\
\hline $\mathrm{ALT}>40 \mathrm{IU} / \mathrm{L}$ & 1.54 & $1.15-2.07$ & 0.005 & 1.35 & $0.98-1.86$ & 0.067 \\
\hline $\mathrm{AFP}>20 \mathrm{ng} / \mathrm{mL}$ & 1.76 & $1.31-2.36$ & $<0.001$ & 1.37 & $1.00-1.87$ & 0.047 \\
\hline $\mathrm{DCP}>40 \mathrm{mAU} / \mathrm{mL}$ & 1.27 & $0.94-1.72$ & 0.119 & & & \\
\hline Red blood cell transfusion & 1.94 & $0.76-4.03$ & 0.152 & & & \\
\hline Surgical margin, positive & 2.69 & $0.95-5.95$ & 0.061 & 1.80 & $0.54-4.50$ & 0.301 \\
\hline Multiple tumor vs. solitary tumor & 2.24 & $1.66-3.00$ & $<0.001$ & 2.22 & $1.64-3.01$ & $<0.001$ \\
\hline Tumor size $>2 \mathrm{~cm}$ & 1.50 & $1.01-2.31$ & 0.042 & 1.72 & $1.15-2.66$ & 0.008 \\
\hline Tumor differentiation, poor ${ }^{\dagger}$ & 1.62 & $0.99-2.52$ & 0.055 & 1.79 & $1.04-2.93$ & 0.037 \\
\hline Vascular invasion, positive & 1.51 & $1.12-20.2$ & 0.007 & 1.47 & $1.08-2.00$ & 0.016 \\
\hline
\end{tabular}

${ }^{*}$ Based on the classification by Desmet et al. (22). $\dagger v s$. well/moderate, based on modification of the Edmondson grade (21). Abbreviations: $\mathrm{HBcAb}$, hepatitis B core antibody; HVCAb, hepatitis C virus antibody; ICG-R15, Indocyanine green retention rate at 15 minutes; ALT, alanine aminotransferase; AFP, $\alpha$-fetoprotein; DCP, des- $\gamma$-carboxyprothrombin.

multivariable analysis revealed that the use of statins reduced the risk of $\mathrm{HCC}$ recurrence after initial liver resection.

Certain drugs, including statins, metformin, and aspirins/NSAIDs, have been reported to alter the risk of HCC development $(11,13,15-17)$. One previous study revealed a protective effect of statin use after liver resection (17). The study demonstrated that in patients with hepatitis B virus-related HCC, the use of nucleoside analogues after liver resection was associated with a significantly lower risk of HCC recurrence. Additionally, use of statins (HR, 0.68) and NSAIDS or aspirins (HR, 0.80 ) were found to be significantly associated with a lower risk of hepatitis B virus-related HCC recurrence. In the present study, use of statins was an independent factor associated with a 0.32 -fold lower risk of HCC recurrence in patients including all etiological backgrounds, whereas an ICG-R15 of $>15 \%$, preoperative ALT concentration of $>40 \mathrm{IU} / \mathrm{L}$, preoperative AFP concentration of $>20$ $\mathrm{ng} / \mathrm{mL}$, preoperative DCP concentration of $>40 \mathrm{mAU} /$ $\mathrm{mL}$, multiple tumors, tumor size of $>2 \mathrm{~cm}$, and vascular invasion were found to be independent risk factors for $\mathrm{HCC}$ recurrence.

The underlying mechanism of the protective effect of statins against the development of HCC has not been fully explained. Some possible mechanisms of 
a

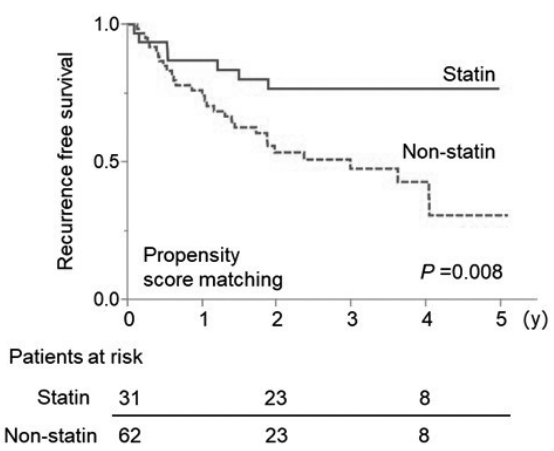

b

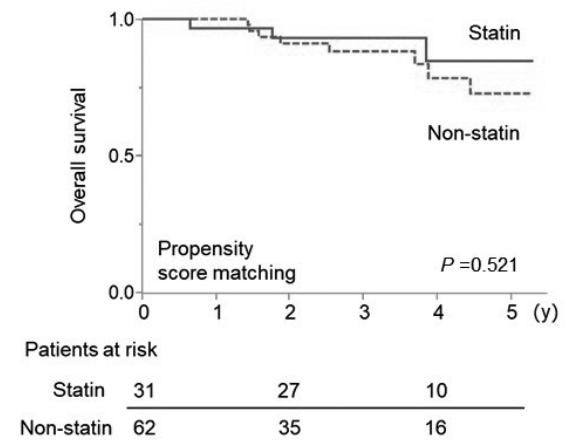

Figure 2. Long-term outcomes between the groups using propensity score-matching analysis. (a) Recurrence-free survival rate was significantly higher in the statin $(n=31)$ than non-statin group $(n=62)(P=0.008)$; (b) Overall survival rate was similar between the groups $(P=0.521)$.

the anticancer effect of statins include inhibition of downstream products of the mevalonate pathway $(33,34)$, triggering of tumor apoptosis (35), inhibition of the proteasome pathway (36), and induction of autophagy (37). Approximately half of patients develop HCC recurrence 3 years after liver resection (2-6). Although adjuvant therapies for HCC have been investigated (7), an effective therapeutic option has not yet been established. Prospective randomized trials are needed to confirm the influence of statin use on patients who have undergone liver resection for $\mathrm{HCC}$.

The main limitation of our study is its retrospective nature and the fact that not all confounders could be completely adjusted for despite the use of a multivariable analysis and a propensity score-matching analysis. The proportion of patients who received statins in our series was small (4.2\%); however, this is in line with the limited proportion of patients who underwent liver resection and received statins $(3.8 \%)$ in a previous report (17). Additionally, the influence of statins was unclear according to each statin type based on the results and the previous studies $(11,13,15-17)$. Second, the protective effects of statins against the development of HCC are not well defined. Additionally, the adverse effects of statins are unclear when they are used for patients without dyslipidemia. Statins are generally contraindicated for patients with liver damage. Finally, we dichotomized continuous variables of potential confounders for HCC prognosis based on previous reports and conducted a multivariable analysis. Such dichotomization may have resulted in lower statistical power.

In conclusion, the risk of HCC recurrence after initial liver resection was lower in patients who received statins than those who did not. Statins may have protective influences on $\mathrm{HCC}$ recurrence in patients who undergo initial liver resection, although further studies are needed to elucidate their adverse effects and influences on HCC recurrence.

\section{Acknowledgements}

This work was supported by by the Program on the Innovative Development and the Application of New Drugs for Hepatitis B from Japan Agency for Medical Research and Development, AMED (to N.K. and K.M.)

\section{References}

1. Jemal A, Bray F, Center MM, Ferlay J, Ward E, Forman D. Global cancer statistics. CA Cancer J Clin. 2011; 61:6990.

2. Belghiti J, Panis Y, Farges O, Benhamou JP, Fekete F. Intrahepatic recurrence after resection of hepatocellular carcinoma complicating cirrhosis. Ann Surg. 1991; 214:114-117.

3. Grazi GL, Ercolani G, Pierangeli F, Del Gaudio M, Cescon M, Cavallari A, Mazziotti A. Improved results of liver resection for hepatocellular carcinoma on cirrhosis give the procedure added value. Ann Surg. 2001; 234:7178.

4. Poon RT, Fan ST, Lo CM, Liu CL, Wong J. Long-term survival and pattern of recurrence after resection of small hepatocellular carcinoma in patients with preserved liver function: implications for a strategy of salvage transplantation. Ann Surg. 2002; 235:373-382.

5. Imamura H, Matsuyama $Y$, Miyagawa $Y$, Ishida K, Shimada R, Miyagawa S, Makuuchi M, Kawasaki S. Prognostic significance of anatomical resection and des-gamma-carboxy prothrombin in patients with hepatocellular carcinoma. Br J Surg. 1999; 86:1032-1038.

6. Nakajima Y, Ko S, Kanamura T, Nagao M, Kanehiro H, Hisanaga M, Aomatsu Y, Ikeda N, Nakano H. Repeat liver resection for hepatocellular carcinoma. J Am Coll Surg. 2001; 192:339-344.

7. Zhu GQ, Shi KQ, Yu HJ, He SY, Braddock M, Zhou MT, Chen YP, Zheng MH. Optimal adjuvant therapy for resected hepatocellular carcinoma: A systematic review with network meta-analysis. Oncotarget. 2015; 6:1815118161.

8. Printz C. Clinical trials of note. Sorafenib as adjuvant treatment in the prevention of disease recurrence in patients with hepatocellular carcinoma (HCC) (STORM). Cancer. 2009; 115:4646.

9. Shirakami Y, Sakai H, Shimizu M. Retinoid roles in blocking hepatocellular carcinoma. Hepatobiliary Surg Nutr. 2015; 4:222-228.

10. Okita K, Izumi N, Ikeda K, et al. Survey of survival among patients with hepatitis C virus-related hepatocellular carcinoma treated with peretinoin, an 
acyclic retinoid, after the completion of a randomized, placebo-controlled trial. J Gastroenterol. 2015; 50:667674.

11. Tsan YT, Lee CH, Wang JD, Chen PC. Statins and the risk of hepatocellular carcinoma in patients with hepatitis B virus infection. J Clin Oncol. 2012; 30:623-630.

12. Hsiang JC, Wong GL, Tse YK, Wong VW, Yip TC, Chan HL. Statin and the risk of hepatocellular carcinoma and death in a hospital-based hepatitis B-infected population: A propensity score landmark analysis. J Hepatol. 2015; 63:1190-1197.

13. Chen CI, Kuan CF, Fang YA, Liu SH, Liu JC, Wu LL, Chang CJ, Yang HC, Hwang J, Miser JS, Wu SY. Cancer risk in HBV patients with statin and metformin use: a population-based cohort study. Medicine (Baltimore) 2015; 94:e462.

14. Butt AA, Yan P, Bonilla H, Abou-Samra AB, Shaikh OS, Simon TG, Chung RT, Rogal SS; ERCHIVES (Electronically Retrieved Cohort of HCV Infected Veterans) Study Team. Effect of addition of statins to antiviral therapy in hepatitis $\mathrm{C}$ virus-infected persons: Results from ERCHIVES. Hepatology. 2015; 62:365-374.

15. Tsan YT, Lee CH, Ho WC, Lin MH, Wang JD, Chen PC. Statins and the risk of hepatocellular carcinoma in patients with hepatitis C virus infection. J Clin Oncol. 2013; 31:1514-1521.

16. Singh S, Singh PP, Singh AG, Murad MH, Sanchez W. Statins are associated with a reduced risk of hepatocellular cancer: A systematic review and meta-analysis. Gastroenterology. 2013; 144:323-332.

17. Wu CY, Chen YJ, Ho HJ, Hsu YC, Kuo KN, Wu MS, Lin JT. Association Between Nucleoside Analogues and Risk of Hepatitis B Virus-Related Hepatocellular Carcinoma Recurrence Following Liver Resection. JAMA. 2012; 308:1906.

18. Makuuchi M, Kosuge T, Takayama T, Yamazaki S, Kakazu T, Miyagawa S, Kawasaki S. Surgery for small liver cancers. Semin Surg Oncol. 1993; 9:298-304.

19. Kubota K, Makuuchi M, Kusaka K, Kobayashi T, Miki K, Hasegawa K, Harihara Y, Takayama T. Measurement of liver volume and hepatic functional reserve as a guide to decision-making in resectional surgery for hepatic tumors. Hepatology. 1997; 26:1176-1181.

20. Kudo M, Kitano M, Sakurai T, Nishida N. General Rules for the Clinical and Pathological Study of Primary Liver Cancer, Nationwide Follow-Up Survey and Clinical Practice Guidelines: The Outstanding Achievements of the Liver Cancer Study Group of Japan. Dig Dis. 2015; 33:765-770.

21. Edmondson HA, Steiner PE. Primary carcinoma of the liver: A study of 100 cases among 48,900 necropsies. Cancer. 1954; 7:462-503.

22. Desmet VJ, Gerber M, Hoofnagle JH, Manns M, Scheuer PJ. Classification of chronic hepatitis: Diagnosis, grading and staging. Hepatology. 1994; 19:1513-1520.

23. Dindo D, Demartines N, Clavien PA. Classification of surgical complications: A new proposal with evaluation in a cohort of 6336 patients and results of a survey. Ann Surg. 2004; 240:205-213.

24. Yamanaka N, Okamoto E, Toyosaka A, Mitunobu M, Fujihara S, Kato T, Fujimoto J, Oriyama T, Furukawa K,
Kawamura E. Prognostic factors after hepatectomy for hepatocellular carcinomas. A univariate and multivariate analysis. Cancer. 1990; 65:1104-1110.

25. Yamamoto J, Kosuge T, Takayama T, Shimada K, Yamasaki S, Ozaki H, Yamaguchi N, Makuuchi M. Recurrence of hepatocellular carcinoma after surgery. Br J Surg. 1996; 83:1219-1222.

26. Lee CS, Sheu JC, Wang M, Hsu HC. Long-term outcome after surgery for asymptomatic small hepatocellular carcinoma. Br J Surg. 1996; 83:330-333.

27. Fuster J, García-Valdecasas JC, Grande L, Tabet J, Bruix J, Anglada T, Taurá P, Lacy AM, González X, Vilana R, Bru C, Solé M, Visa J. Hepatocellular carcinoma and cirrhosis. Results of surgical treatment in a European series. Ann Surg. 1996; 223:297-302.

28. Fan ST, Ng IO, Poon RT, Lo CM, Liu CL, Wong J. Hepatectomy for hepatocellular carcinoma: the surgeon's role in long-term survival. Arch Surg. 1999; 134:11241130.

29. Vauthey JN, Lauwers GY, Esnaola NF, Do KA, Belghiti J, Mirza N, Curley SA, Ellis LM, Regimbeau JM, Rashid A, Cleary KR, Nagorney DM. Simplified staging for hepatocellular carcinoma. J Clin Oncol. 2002; 20:15271536.

30. Ikeda K, Marusawa H, Osaki Y, Nakamura T, Kitajima N, Yamashita Y, Kudo M, Sato T, Chiba T. Antibody to hepatitis B core antigen and risk for hepatitis C-related hepatocellular carcinoma: a prospective study. Ann Intern Med. 2007; 146:649-656.

31. Austin PC. Statistical criteria for selecting the optimal number of untreated subjects matched to each treated subject when using many-to-one matching on the propensity score. Am J Epidemiol. 2010;172:1092-1097.

32. D'Agostino RB, Jr. Propensity score methods for bias reduction in the comparison of a treatment to a nonrandomized control group. Stat Med. 1998; 17:2265-2281.

33. Danesh FR, Sadeghi MM, Amro N, Philips C, Zeng L, Lin S, Sahai A, Kanwar YS. 3-Hydroxy-3-methylglutaryl CoA reductase inhibitors prevent high glucose-induced proliferation of mesangial cells via modulation of Rho GTPase/ p21 signaling pathway: Implications for diabetic nephropathy. Proc Natl Acad Sci U S A. 2002;99:83018305.

34. Wong WW, Dimitroulakos J, Minden MD, Penn LZ. HMG-CoA reductase inhibitors and the malignant cell: The statin family of drugs as triggers of tumor-specific apoptosis. Leukemia. 2002; 16:508-519.

35. Chan KK, Oza AM, Siu LL. The statins as anticancer agents. Clin Cancer Res. 2003; 9:10-19.

36. Rao S, Porter DC, Chen X, Herliczek T, Lowe M, Keyomarsi K. Lovastatin-mediated G1 arrest is through inhibition of the proteasome, independent of hydroxymethyl glutaryl-CoA reductase. Proc Natl Acad Sci U S A. 1999; 96:7797-7802.

37. Yang PM, Liu YL, Lin YC, Shun CT, Wu MS, Chen CC. Inhibition of autophagy enhances anticancer effects of atorvastatin in digestive malignancies. Cancer Res. 2010; 70:7699-7709.

(Received August 20, 2017; Revised October 17, 2017; Accepted October 18, 2017) 